\title{
Binge-Like Consumption of Ethanol and Other Salient Reinforcers is Blocked by Orexin-1 Receptor Inhibition and Leads to a Reduction of Hypothalamic Orexin Immunoreactivity
}

\author{
Jeffrey J. Olney, Montserrat Navarro, and Todd E. Thiele \\ Department of Psychology (JJO, MN, TET), University of North Carolina, Chapel Hill, North \\ Carolina; and Bowles Center for Alcohol Studies (TET), University of North Carolina, Chapel Hill, \\ North Carolina
}

\begin{abstract}
Background-Orexin (OX) neurons originating in the lateral hypothalamus (LH) are ideally positioned to modulate reward processing as they form connections with several key brain regions known to be involved in the reward pathway. Consistent with these findings, a growing number of studies have implicated the OX system in modulating the rewarding properties of several drugs of abuse, including ethanol (EtOH). However, the role of the OX system in excessive binge-like EtOH intake remains relatively unexplored. Here, we assessed changes in OX immunoreactivity (IR) in the hypothalamus following repeated cycles of binge-like EtOH drinking and assessed the participation of the OX-1 receptor (OX1R) in binge-like EtOH consumption.
\end{abstract}

Methods-The drinking-in-the-dark (DID) paradigm was used to model binge-like EtOH drinking in male C57BL/6J mice. In the first experiment, mice experienced 1 or 3 cycles of bingelike EtOH or sucrose drinking with DID procedures to assess changes in OX IR in distinct subregions of the hypothalamus. Subsequent experiments examined binge-like EtOH and saccharin drinking following peripheral injections of 0.0, 5.0, or $10.0 \mathrm{mg} / \mathrm{kg}$ SB-334867 (SB), a selective OX1R antagonist. Finally, mice were given peripheral injections of SB and open-field locomotor activity was measured.

Results-Relative to water drinking controls, binge-like consumption of EtOH and sucrose resulted in a marked reduction in OX IR in the LH. Inhibition of the OX1R via SB blunted EtOH and saccharin drinking, but did not alter open-field locomotor activity.

Conclusions-Our observed reduction in OX IR in the LH indicates that the OX system in engaged during binge-like consumption of EtOH and sucrose. The observation that inhibition of the OX1R signaling blunted binge-like EtOH, and saccharin drinking suggests that reward-related

Copyright () 2015 by the Research Society on Alcoholism.

Reprint requests: Todd E. Thiele, PhD, Department of Psychology, University of North Carolina, Davie Hall, CB \#3270, Chapel Hill, NC 27599-3270; Tel.: 919-966-1519; Fax: 919-962-2537; thiele@ unc.edu.

SUPPORTING INFORMATION

Additional Supporting Information may be found in the online version of this article:

Data S1. Orexin-A Immunohistochemical Analysis.

Data S2. Locomotor Testing. 
OX circuits originating in the LH participate in the consumption of salient reinforcers regardless of calories.

\section{Keywords}

Binge-Like Ethanol Consumption; Drinking in the Dark; Orexin; Hypocretin; Orexin-1 Receptor

CENTRAL OREXIN (OX) is synthesized exclusively within the hypothalamus and is involved in a host of neurobiological functions, including feeding (Sakurai et al., 1998), stress (Winsky-Sommerer et al., 2004), arousal (Chemelli et al., 1999), and, more recently, reward-related behavior (Harris et al., 2005). It is believed that the functional responsibilities of central OX neurons are segregated within the hypothalamus. Specifically, lateral hypothalamic (LH) OX neurons have been proposed to be more involved in reward-related behaviors (Harris et al., 2005), while OX neurons from the perifornical area of the hypothalamus (PFA) and dorsomedial hypothalamus (DMH) are more associated with stress and arousal (Harris and Aston-Jones, 2006). Although OX originates solely within the hypothalamus (Sakurai et al., 1998), its afferents form connections with regions across the brain (Yoshida et al., 2005). Consistent with these findings, OX projections originating from the LH facilitate the activity of several brain regions in the reward circuit, such as the ventral tegmental area (VTA; Borgland et al., 2006; Korotkova et al., 2003; Moorman and AstonJones, 2010) and shell of the nucleus accumbens (NAc; Patyal et al., 2012).

In agreement with the widespread connections to various regions in the reward circuit, OX has been found to be involved in the neurobiological responses to ethanol (EtOH). Lawrence and colleagues (2006) first observed this relationship by systemically injecting the selective OX-1 receptor (OX1R) antagonist, SB-334867 (SB) to reduce operant self-administration of EtOH. Similarly, SB was found to selectively reduce EtOH consumption in high-, but not low-, EtOH-preferring rats (Moorman and Aston-Jones, 2009). Further investigations suggest that modulation of OX signaling via SB is specific to EtOH as Jupp and colleagues (2011) found that SB significantly attenuated the motivational effects of EtOH but not sucrose. Although these data suggest an essential role for OX1R signaling in EtOH drinking, recent evidence suggests the $\mathrm{OX} 2 \mathrm{R}$ is also capable of modulating responses to $\mathrm{EtOH}$ (Anderson et al., 2014; Barson et al., 2014; Shoblock et al., 2011). Moreover, more comprehensive investigations of the role of $\mathrm{OX}$ in EtOH drinking have revealed that signaling within the reward-related pathway is responsible for modulating this behavior. Indeed, direct infusions of orexin-A in the LH (Schneider et al., 2007) and a nonselective OXR antagonist into the VTA (Srinivasan et al., 2012) significantly increased and decreased EtOH drinking, respectively. As a whole, these findings indicate EtOH drinking directly parallels OX signaling in the reward pathway.

In addition to the $\mathrm{OX}$ system modulating the neurobiological responses to $\mathrm{EtOH}$, it has previously been reported that EtOH experience produces alterations in the OX system. Indeed, chronic EtOH drinking increased OX mRNA, as measured by in situ hybridization (ISH), in the LH relative to EtOH naïve rats (Lawrence et al., 2006). Interestingly, Morganstern and colleagues (2010) reported that chronic EtOH consumption caused a significant reduction in hypothalamic OX mRNA, as measured by quantitative real-time 
polymerase chain reaction (qRT-PCR), while acute EtOH exposure via oral gavage significantly enhanced OX mRNA and orexin-A levels, as measured by ISH and immunohistochemistry (IHC), respectively. What is more, Barson and colleagues (2014) recently used qRT-PCR to assess changes in OX mRNA following EtOH drinking in an intermittent-access paradigm and found increased OX expression in the hypothalamus. Although these findings demonstrate experience with EtOH alters central OX levels, different methodological approaches, such as acute versus chronic and self-versus experimenter-administered $\mathrm{EtOH}$, may influence the nature of this effect.

Despite this growing body of literature implicating the OX system in EtOH consumption, relatively less is known of its role in binge drinking behavior. Considering recent evidence that indicates different neurocircuitry may be engaged during binge-like versus moderate levels of EtOH drinking (Lowery et al., 2010; Lowery-Gionta et al., 2012; Sparta et al., 2008), the goal of this study was to further characterize the role of the central OX system in binge-like drinking behavior. To this end, we first examined the influence of repeated cycles of binge-like EtOH or sucrose drinking on hypothalamic OX levels using IHC procedures.

Additionally, we peripherally administered the selective OX1R antagonist, SB, to characterize the participation of the OX1R in binge-like EtOH consumption, and we assessed the specificity of SB in modulating EtOH drinking by examining its effects on saccharin, a salient but noncaloric reinforcer. Finally, we also examined the effects of this pharmacological inhibition on more general behavioral responses by assessing locomotor activity following treatment with SB.

\section{MATERIALS AND METHODS}

\section{Animals}

A total of 90 male C57BL/6J (C57) mice (Jackson Laboratories, Bar Harbor, ME) aged 7 to 9 weeks were used in this study. Mice were individually housed in plastic cages located in a vivarium with an ambient temperature of approximately $22^{\circ} \mathrm{C}$ and a reverse light/dark cycle with lights off at 7:00 Ам. Food and water were available ad libitum except during testing (see below). All procedures used were in accordance with the National Institutes of Health guidelines and were approved by the University of North Carolina Institutional Animal Care and Use Committee.

\section{OX Immunoreactivity Following Repeated Cycles of Binge-Like EtOH Intake}

Fifty C57 mice experienced 1 or 3 cycles of binge-like EtOH drinking using the "drinkingin-the-dark" (DID) procedure. The DID procedure is a commonly used animal model of binge-like EtOH drinking that promotes high levels of consumption and generates physiologically relevant blood EtOH concentrations (BECs) of $80 \mathrm{mg} / \mathrm{dl}$ or greater (Rhodes et al., 2005, 2007). On days 1 to 3 , standard water bottles were removed 3 hours into the dark cycle and the animals were given access to test bottles containing water, EtOH (20\% $\mathrm{v} / \mathrm{v})$, or sucrose $(3 \% \mathrm{w} / \mathrm{v})$ solutions for 2 hours. Binge-like consumption was assessed on the fourth day when access to the test bottles was extended to 4 hours. Each 4-day test period constituted a binge cycle, and there were 3 days with no EtOH access between binge cycles. 
The start dates of the binging cycles were staggered such that all groups finished testing on the same day and at the approximate same age.

Refer to the Supplementary Materials for a complete description of the IHC procedure. Briefly, on the final day of testing, tail bloods were collected to assess BECs and brains were collected and processed for orexin-A immunoreactivity (IR). The number of hypothalamic orexin-A positive neurons was quantified and grouped based on hypothalamic subregion (Fig. $2 A$; i.e., LH, PFA, or DMH) as defined previously (Mahler and Aston-Jones, 2012). Upon quantifying levels of orexin-A positive cells in our animals, it became clear that the DMH exhibited minimal orexin-A IR in our samples (grand mean: 3.327, SEM: 0.352); therefore, this subregion was removed from further analysis.

Univariate analyses of variance (ANOVAs) were used to measure orexin-A positive cells in the LH and PFA separately using group (water, 1-cycle EtOH, 1-cycle sucrose, 3-cycle $\mathrm{EtOH}$, or 3-cycle sucrose) as the independent variable. The brain tissue from an animal in the 3-cycle EtOH group was damaged during the staining process making it unable to be quantified and was removed from the analysis. To ensure that hypothalamic OX expression was not affected by differential drinking levels, separate $t$-tests were used to assess consumption of each test solution (i.e., EtOH or sucrose) on the final day of testing between animals in the 1 or 3 DID cycle groups. Similarly, a $t$-test was also performed on the BEC data to measure differences in EtOH metabolism as a function of DID cycles (1 or 3). Tukey's HSD post hoc tests and Bonferroni corrections were used when applicable.

\section{Binge-Like EtOH Drinking Following Treatment with SB}

A separate cohort of $20 \mathrm{C} 57$ mice was used to assess the effect of pharmacological inhibition of OX1R on binge-like consumption of $20 \% \mathrm{EtOH}(\mathrm{v} / \mathrm{v})$. Initial investigations utilized the standard DID procedure as described above with the addition that consumption was recorded every hour during testing to assess the drug's effect over time. However, early observations indicated that the effect of the pharmacological treatment was short-lived (data not shown). Therefore, to better capture the transient effects of the drug, subsequent iterations used a modified DID paradigm that was identical to the standard DID procedure described above with the exception that the animals only had access to the EtOH bottles for 2 hours on the fourth day. All other test parameters remained unchanged.

Thirty minutes prior to $\mathrm{EtOH}$ access on the fourth day, mice were given intraperitoneal (i.p.) injections of $0.0,5.0$, or $10.0 \mathrm{mg} / \mathrm{kg} \mathrm{SB}$ (Tocris Bioscience, Minneapolis, MN), which was dissolved using $0.01 \%$ Tween ${ }^{\circledR} 80$ (Sigma-Aldrich, St. Louis, MO) in saline as described previously (Anderson et al., 2014). All doses were administered in a $10.0 \mathrm{ml} / \mathrm{kg}$ injection volume. Immediately after testing, tail-blood samples were collected from each animal and analyzed as described above. To increase power during statistical analysis, each animal received all 3 doses of the drug over repeated trials to allow for within-subject comparison of EtOH drinking behavior. Mice were given 3 days rest between subsequent 4-day DID sessions to avoid carryover effects of the drug.

A repeated-measures ANOVA was used to assess hourly EtOH consumption with both time (hour 1 and hour 2) and SB dose $(0.0,5.0$, or $10.0 \mathrm{mg} / \mathrm{kg})$ being within-subject variables. 
Additionally, total EtOH consumption across the 2-hour test period was assessed using a repeated-measures ANOVAs with SB dose $(0.0,5.0$, or $10.0 \mathrm{mg} / \mathrm{kg})$ as the within-subject variable. As the first round of DID in the within-subjects design followed the standard, 4hour DID model, the tail-blood samples from the first round were excluded from the analysis. Due to the exclusion of these data points, a univariate ANOVA was used to assess BECs with dose of SB $(0.0,5.0$, or $10.0 \mathrm{mg} / \mathrm{kg})$ as the independent variable. Additionally, drug order was included as a between-subjects variable in these analyses to ensure the order in which the animals were presented the drug did not have any confounding effects on $\mathrm{EtOH}$ consumption. Tukey's HSD post hoc tests and Bonferroni corrections were used when applicable.

\section{Binge-Like Saccharin Drinking Following Treatment with SB}

A separate cohort of 20 C57 mice was used to assess binge-like consumption of saccharin following treatment with SB. The procedures used here were identical to those described above except that these mice were given access to saccharin $(0.15 \% \mathrm{w} / \mathrm{v})$ during the modified, 2-hour DID procedure. A Latin square design was used such that each animal received all 3 doses of the drug over repeated trials. Mice were given at least 3 days of rest between subsequent 4-day DID sessions to avoid carryover effects of the drug.

A repeated-measures ANOVA was used to assess hourly saccharin consumption with both time (hours 1 and 2 ) and dose of SB $(0.0,5.0$, or $10.0 \mathrm{mg} / \mathrm{kg}$ ) being within-subject variables. Total saccharin consumption was also assessed using separate repeated-measures ANOVAs with SB dose $(0.0,5.0$, or $10.0 \mathrm{mg} / \mathrm{kg})$ as the within-subject variable. Drug order was also included as a between-subjects variable in these analyses. Tukey's HSD post hoc tests and Bonferroni corrections were used when applicable.

\section{Locomotor Activity Following Treatment with SB}

The same 20 mice from the previous saccharin experiment were used to assess locomotor activity following treatment with SB. The complete procedure for locomotor testing can be found in the Supplementary Materials section. Briefly, 1 week following saccharin testing, animals were given i.p. injections of 0.0 or $10.0 \mathrm{mg} / \mathrm{kg}$ SB as described above. Thirty minutes later, animals were placed in an open-field locomotor chamber and 2-hour locomotor activity was measured. The decision to use only the $10.0 \mathrm{mg} / \mathrm{kg}$ dose of SB was based on the findings from our previous experiment that only the higher dose produced significant reductions in EtOH consumption. Unlike our previous pharmacological experiments, a Latin square design was not used to avoid previous experience with the locomotor chamber confounding the animals' locomotor activity.

Separate t-tests were used to assess the effects of SB on locomotor activity. In addition to measuring the effects of SB across the entire 2-hour test period, the immediate effects of the drug during the first 15 minutes of testing were also assessed. 


\section{RESULTS}

\section{Binge-Like Consumption of a Salient Reinforcer Reduces OX Levels}

Animals' responses to the test solutions were comparable regardless of DID history as EtOH and sucrose drinking on the final day of testing did not differ between animals that experienced 1 cycle or 3 cycles of DID (Fig. $1 A, B ; t(18)=0.1316, p=0.897 ; t(18)=0.4746$, $p=0.641$, respectively). Moreover, both groups of EtOH drinkers achieved similar BECs regardless of DID history (Fig. $1 C ; t(18)=0.603, p=0.5540$ ). This allows us to make direct comparisons of IR between the different groups without the potential confound of group differences in brain-EtOH exposure.

Our analysis revealed that binge-like consumption of EtOH or sucrose solutions significantly altered the number of orexin-A positive neurons within the LH (Figs $1 D$ and $2 B-F), F(4,48)=5.863, p=0.001$. Further probing of this effect revealed that relative to the water group (Fig. $2 D$ ), all test groups (i.e., 1-cycle EtOH [Fig. 2B], 3-cycle EtOH [Fig. $2 C$ ], 1-cycle sucrose [Fig. 2E], and 3-cycle sucrose [Fig. 2F]) displayed significantly reduced OX levels (Fig. $1 D$; $p s<0.010$ ). Similarly, binge-like consumption of EtOH or sucrose also significantly altered OX levels within the PFA (Fig. $2 B-F$ ), $F(4,48)=3.702, p$ $=0.011$. Unlike the LH, however, Tukey's HSD tests indicated that only the 3-cycle EtOH group showed significant reductions in OX expression in the PFA relative to the water group (Fig. $1 D ; p=0.006$ ).

\section{Treatment with SB Attenuates Binge-Like EtOH Consumption}

The previous experiment demonstrated that binge-like EtOH drinking affects hypothalamic OX levels. Next, we sought to determine whether manipulating OX signaling via the selective OX1R antagonist, SB, could modulate binge-like EtOH drinking. As shown in Fig. $3 A$, treatment with SB significantly reduced binge-like EtOH consumption (main effect of dose: $F(2,38)=6.083, p=0.005)$, but the animals' drinking behavior did not change over time (main effect of time: $F(1,19)=0.372, p=0.549$ ). Analysis also showed that there was a significant drug by time interaction effect, $F(2,38)=8.196, p=0.001$. Further probing revealed both the 5.0 and $10.0 \mathrm{mg} / \mathrm{kg}$ dose of SB significantly reduced binge-like EtOH drinking during the first hour relative to water ( $p s<0.05$ ), although the higher dose of SB significantly reduced binge-like EtOH drinking to a level beyond the lower dose $(p=0.015)$. Importantly, the effect of drug order was not significant, $F(2,17)=0.862, p=0.481$, indicating that the order in which the animals received the SB treatment did not affect hourly EtOH drinking behavior. Additionally, SB significantly reduced EtOH drinking over the 2hour test period, $F(2,38)=9.870, p<0.001$. Interestingly, the effect of the lower dose of SB appeared to be short-lived as only the higher dose significantly blunted EtOH drinking over the full 2 hours $(p<0.001)$. This effect of the higher dose across the 2-hour test period appears to be driven by the effect at the first hour as no differences were observed during the second hour of testing. Similar to the hourly consumption, drug order did not impact total EtOH drinking behavior, $F(2,17)=0.862, p=0.481$.

In accordance with the drinking data, treatment with SB significantly reduced measured BECs as well (Fig. $3 B), F(2,59)=4.312, p=0.018$. Further probing of this effect indicated 
animals treated with the higher dose of SB exhibited significantly lower BECs relative to vehicle treated animals $(p=0.013)$. Finally, order of drug presentation did not affect measured BECs, $F(1,56)=0.729, p=0.539$.

\section{Treatment with SB Attenuates Binge-Like Saccharin Consumption}

The previous experiment demonstrated that pharmacological inhibition of the OX1R is capable of reducing excessive EtOH consumption in a DID paradigm. Interestingly, a recent study by Anderson and colleagues (2014) observed that systemic treatment with SB reduces binge-like sucrose consumption, which suggests the OX system modulates binge-like consumption of general, salient reinforcers beyond EtOH. However, as sucrose and EtOH are both reinforcing sources entailing caloric properties, it remains to be seen whether or not these effects are due alterations in caloric need. Thus, the next experiment sought to characterize the effects of SB on saccharin, a salient, noncaloric reinforcer.

Analysis of hourly intake revealed SB significantly reduced saccharin consumption (Fig. 4; main effect of dose: $F(2,38)=4.231, p=0.022)$. Further probing of this main effect showed only the higher dose $(10.0 \mathrm{mg} / \mathrm{kg})$ of SB significantly attenuated saccharin intake after Bonferroni's correction $(p=0.011)$. A significant main effect of time indicated all animals drank more saccharin during the first hour than the second (main effect of time: $F(1,38)=$ $11.756, p=0.003)$. The dose by time interaction effect did not reach significance, $F(2,38)=$ $1.089, p=0.347$. Additionally, SB significantly reduced total saccharin intake across the full 2 -hour test period, $F(2,38)=4.231, p=0.022$. Similar to the hourly consumption, follow-up tests revealed only the higher dose of SB significantly attenuated saccharin intake after Bonferroni's correction $(p=0.011)$. As with the previous experiment, drug order did not affect hourly, $F(2,17)=0.024, p=0.977$ or total, $F(2,17)=0.018, p=0.982$ saccharin drinking behavior. Together, findings from this experiment indicate the effect of reduced binge-like drinking following pharmacological inhibition of the OX1R via SB is not specific to EtOH but appears to blunt consumption of salient caloric $(\mathrm{EtOH})$ and noncaloric (saccharin) reinforcers.

\section{Treatment with SB Does Not Affect Locomotor Activity}

Perhaps one of the most widely studied functions of the OX system is its role in sleep and arousal (for review, see De Lecea, 2012). To rule out the possibility that our observed effects of SB on EtOH or saccharin consumption could be secondary to the effects of SB on general motor behavior, the next experiment sought to test the effects of SB on open-field locomotor activity. Simple $t$-tests showed that, relative to vehicle, treatment with the 10.0 $\mathrm{mg} / \mathrm{kg}$ dose of SB had no discernable effect on immediate locomotor activity during the first 15 minutes of testing, $t(18)=0.2129, p=0.834$, nor across the entire 2-hour test period, $t(18)=1.630, p=0.1204$ (Fig. 5). Together, these data demonstrate that our observed effects of SB on the consumption of salient reinforcers are not a consequence of impaired locomotor activity. 


\section{DISCUSSION}

Through our series of experiments, we demonstrated that binge-like consumption of a salient reinforcer using the DID model resulted in a marked reduction of hypothalamic OX IR. Similarly, blocking the OX1R signaling via SB protects against excessive EtOH consumption. Interestingly, this effect is not specific to EtOH as treatment with SB also reduced binge-like consumption of saccharin; however, no other nonspecific effects were observed as SB did not significantly alter locomotor activity.

The observations made here confirm and extend several findings reported previously. First, exposure to EtOH has previously been shown to alter hypothalamic OX peptide IR and mRNA (Lawrence et al., 2006; Morganstern et al., 2010). Our experiments demonstrate that binge-like consumption of EtOH reduces the number orexin-A IR neurons in the hypothalamus. Interestingly, these effects were not specific to EtOH as a similar pattern of effects was found in animals that experienced binge-like sucrose consumption. Moreover, these effects were most prominent in the $\mathrm{LH}$, a subregion of the hypothalamus believed to be more strongly associated with reward-related behaviors (Harris et al., 2005). Further, our animals showed minimal changes in OX IR levels in the PFA following binge-like consumption of a salient reinforcer, a subregion postulated to be more involved in stress and arousal (Harris and Aston-Jones, 2006). Although our data indicated mice that experienced 3 cycles of binge-like EtOH consumption showed reductions in OX IR in the PFA, this effect may be due to the fact that EtOH impacts normal sleep patterns (Ebrahim et al., 2013). Indeed, EtOH has been shown to promote sleep by inhibiting OX neurons in the PFA (Sharma et al., 2014). Together, these data provide support for the dichotomy of OX function between the LH and PFA.

Previous pharmacological studies have reported that EtOH consumption parallels OX signaling (Jupp et al., 2011; Lawrence et al., 2006; Moorman and Aston-Jones, 2009; Schneider et al., 2007). In light of these reports, we believe our observed reduction in LH OX IR reflects an increase in OX signaling. In this case, OX would be released from its presynaptic stores in response to consumption of salient reinforcers. As our IHC analysis measured the amount of peptide immediately following the last round of DID, a reduction in OX IR may be indicative of an increase in OX signaling although further testing, such as changes in OX mRNA following DID, is required to confirm this hypothesis. This finding would be consistent with previous observations that acute EtOH exposure causes a marked increase in OX mRNA and orexin-A peptide (Barson et al., 2014; Morganstern et al., 2010). Interestingly, it was also observed that chronic voluntary EtOH drinking causes a reduction in OX mRNA (Morganstern et al., 2010); thus, future studies would benefit from examining changes in OX following chronic binge-like EtOH drinking. Alternatively, a reduction in observed OX levels may reflect a compensatory reduction in the system, which has recently been observed following binge-like sucrose and saccharin drinking (Alcaraz-Iborra et al., 2014).

Additionally, we observed that selective inhibition of the OX1R protected against excessive EtOH consumption using the DID procedure. This observation confirms several others that have reported that prior treatment with SB significantly reduces responding to EtOH in a 
variety of paradigms (Anderson et al., 2014; Jupp et al., 2011; Moorman and Aston-Jones, 2009; Voorhees and Cunningham, 2011). Notably, our mice exhibited robust EtOH intake, yet the BEC levels were slightly below binge criteria that may stem from the shortened 2hour test. What is more, our findings indicate the protective effect of SB is not specific to $\mathrm{EtOH}$ as we also observed that SB reduced excessive intake of saccharin, a noncaloric reinforcer. In fact, it has recently been reported that systemic SB protects against binge-like EtOH and sucrose intake (Alcaraz-Iborra et al., 2014; Anderson et al., 2014). Together with our observation that peripherally administered SB similarly blunts EtOH and saccharin drinking, these findings suggest the OX1R modulates the consumption of salient reinforcers regardless of caloric content. Although previous findings have demonstrated that the protective effect of SB is specific to EtOH and not sucrose (Jupp et al., 2011), this difference could be attributed to procedural differences. That is, the prior study used operant responding to $\mathrm{EtOH}$ and sucrose in alcohol-preferring (iP) rats, while the present study used the DID procedure in C57 mice.

Moreover, the recent report by Anderson and colleagues (2014) described similar success in using SB to significantly reduce binge-like EtOH drinking in C57s using the standard DID model. Interestingly, that same report found that only a high dose of SB $(30.0 \mathrm{mg} / \mathrm{kg})$ reduced binge-like EtOH drinking, while lower doses were ineffective. Although the effective dose of SB between this previous report and our current study is at variance with one another, there are methodological differences that may be able to explain this disparity. First, a great deal of variability in SB has been documented between different vendors and even different batches of the same vendor (Jupp et al., 2011; Mahler et al., 2012). The previous study used SB that was synthesized by one of the researchers at Lilly Research Laboratories, while we ordered ours from a private vendor. This may be of importance as the manner in which the compound is synthesized may alter its effectiveness (McElhinny et al., 2012). Therefore, the possibility that the different effective dose observed between our studies is due to some inherent difference between the 2 SB compounds cannot be ruled out.

Another difference between these 2 reports is the time intervals at which consumption data were measured. The previous study reported that only the $30.0 \mathrm{mg} / \mathrm{kg}$ dose of SB was effective in significantly reducing EtOH drinking over a 4-hour test period. However, our preliminary data using a lower dose suggested the effect of SB did not persist throughout the entire 4-hour test period (pilot data not shown) and prompted us to shorten the test period as well as take hourly measures to assess drinking over time. Using this design, we were able to discover that lower doses are indeed capable of reducing excessive EtOH consumption although the effect is short-lived.

Although this report demonstrates that inhibition of the OX system protects against bingelike EtOH consumption, the systemic nature of our treatment procedure is unable to determine the locus of this effect. Future studies will attempt to identify the OX circuits that convey these effects via site-specific delivery of OX agents directly to candidate regions. The LH is known to be involved in reward-related behavior (DiLeone et al., 2003; Wise, 1996), and OX neurons originated from the LH have been reported to project to key brain regions in the reward circuit (Marcus et al., 2001; Yoshida et al., 2005). Both the NAc shell and VTA are densely innervated by OX neurons and are known to be involved in reward- 
related behavior. Indeed, application of OX peptide causes a robust depolarization of the neurons within the shell of the NAc in vitro (Mukai et al., 2009). However, direct infusion of orexin-A into the NAc shell failed to alter EtOH consumption (Schneider et al., 2007) suggesting that $\mathrm{OX}$ signaling in this brain region may not modulate EtOH drinking behavior. On the other hand, it has been previously demonstrated that application of orexin-A alone initiates plastic changes in the VTA that facilitates activity in this region (Borgland et al., 2006). Moreover, blocking OXRs in the VTA has been shown to protect against EtOH selfadministration (Srinivasan et al., 2012). Taken together, these findings suggest the OX neurons originating in the LH that project to the VTA may be a key circuit that modulates EtOH drinking.

Interestingly, recent evidence indicates behaviors such as motivation and reward are modulated by the interplay between OX and dynorphin signaling within the VTA. These 2 neuropeptides, which have been shown to be expressed in the same presynaptic vesicles, may have an oppositional relationship such that the relative signaling between these 2 peptides modulates behavior (Muschamp et al., 2014). In light of this recent discovery, our observed reduction in binge-like drinking behavior may be due not to a reduction in OX1R signaling, per se, but a reduction in OX1R signaling relative to dynorphin signaling. Although our manipulations were specific to the OX system, our assays are unable to speak to the state of the dynorphin system. Regardless of the activity in the dynorphin system, however, our data demonstrate that the OX system is engaged during binge-like EtOH drinking and suppressing signaling within this system blunts this behavior.

Finally, as the OX system is known to be critically involved in sleep and arousal (Chemelli et al., 1999), it was important to rule out the possibility that our observed effects of SB on responding to salient reinforcers were not due to an induction of a hypo-active state. Given that treatment with SB did not alter locomotor activity at any time point measured, it is unlikely that inhibiting the OX1R could have produced any effects on arousal that could have confounded our effects on binge-like consummatory behavior. However, our results on locomotor activity cannot rule out the possibility that SB may induce a state of anhedonia and/or amotivation. Indeed, a reduction in OX levels in the VTA and hypothalamus was observed in a rat social defeat model of depression (Nocjar et al., 2012). Future studies will explore the induction of a state of anhedonia and/or amotivation as a possible cause for reduced binge-like EtOH drinking.

Findings from the present report indicate that LH OX levels are blunted following binge-like consumption of EtOH and sucrose. Moreover, pharmacological inhibition of signaling onto the OX1R served to protect against excessive EtOH and saccharin consumption without altering locomotor activity. Together, these findings provide promise that targeting the OX system through OXR antagonists may serve as an effective treatment for alcohol use disorders.

\section{Supplementary Material}

Refer to Web version on PubMed Central for supplementary material. 


\section{ACKNOWLEDGMENTS}

This work was supported by National Institutes of Health grants AA013573, AA015148, and AA022048.

\section{REFERENCES}

Alcaraz-Iborra M, Carvajal F, Lerma-Cabrera JM, Valor LM, Cubero I. Binge-like consumption of caloric and non-caloric palatable substances in ad libitum-fed C57BL/6J mice: pharmacological and molecular evidence of orexin involvement. Behav Brain Res. 2014; 272:93-99. [PubMed: 24983661]

Anderson RI, Becker HC, Adams BL, Jesudason CD, Rorick-Kehn LM. Orexin-1 and orexin-2 receptor antagonists reduce ethanol self-administration in high-drinking rodent models. Front Neurosci. 2014; 8:33. [PubMed: 24616657]

Barson JR, Ho HT, Leibowitz SF. Anterior thalamic paraventricular nucleus is involved in intermittent access ethanol drinking: role of orexin receptor 2. Addict Biol. 2014 doi: 10.1111/adb.12139 [Epub ahead of print].

Borgland S, Taha SA, Sarti F, Fields HL, Bonci A. Orexin A in the VTA is critical for the induction of synaptic plasticity and behavioral sensitization to cocaine. Neuron. 2006; 49:589-601. [PubMed: 16476667]

Chemelli RM, Willie JT, Sinton CM, Elmquist JK, Scammell T, Lee C, Richardson JA, Williams SC, Xiong Y, Kisanuki Y, Fitch TE, Nakazato M, Hammer RE, Saper CB, Yanagisawa M. Narcolepsy in orexin knockout mice: molecular genetics of sleep regulation. Cell. 1999; 98:437-451. [PubMed: 10481909]

De Lecea, L. Hypocretins and the neurobiology of sleep-wake mechanisms. In: Shekhar, A., editor. Progress in Brain Research. Elsevier; 2012. p. 15-24.Available at: http://linkinghub.elsevier.com/ retrieve/pii/B9780444594891000033 [Accessed June 18, 2014]

DiLeone RJ, Georgescu D, Nestler EJ. Lateral hypothalamic neuropeptides in reward and drug addiction. Life Sci. 2003; 73:759-768. [PubMed: 12801597]

Ebrahim IO, Shapiro CM, Williams AJ, Fenwick PB. Alcohol and sleep I: effects on normal sleep. Alcohol Clin Exp Res. 2013; 37:539-549. [PubMed: 23347102]

Harris GC, Aston-Jones G. Arousal and reward: a dichotomy in orexin function. Trends Neurosci. 2006; 29:571-577. [PubMed: 16904760]

Harris GC, Wimmer M, Aston-Jones G. A role for lateral hypothalamic orexin neurons in reward seeking. Nature. 2005; 437:556-559. [PubMed: 16100511]

Jupp B, Krivdic B, Krstew E, Lawrence AJ. The orexin1 receptor antagonist SB-334867 dissociates the motivational properties of alcohol and sucrose in rats. Brain Res. 2011; 1391:54-59. [PubMed: 21439948]

Korotkova TM, Sergeeva OA, Eriksson KS, Haas HL, Brown RE. Excitation of ventral tegmental area dopaminergic and nondopaminergic neurons by orexins/hypocretins. J Neurosci. 2003; 23:7-11. [PubMed: 12514194]

Lawrence AJ, Cowen MS, Yang HJ, Chen F, Oldfield B. The orexin system regulates alcohol-seeking in rats. Br J Pharmacol. 2006; 148:752-759. [PubMed: 16751790]

Lowery EG, Spanos M, Navarro M, Lyons AM, Hodge CW, Thiele TE. CRF-1 antagonist and CRF-2 agonist decrease binge-like ethanol drinking in C57BL/6J mice independent of the HPA axis. Neuropsychopharmacology. 2010; 35:1241-1252. [PubMed: 20130533]

Lowery-Gionta EG, Navarro M, Li C, Pleil KE, Rinker JA, Cox BR, Sprow GM, Kash TL, Thiele TE. Corticotropin releasing factor signaling in the central amygdala is recruited during binge-like ethanol consumption in C57BL/6J mice. J Neurosci. 2012; 32:3405-3413. [PubMed: 22399763]

Mahler SV, Aston-Jones GS. Fos activation of selective afferents to ventral tegmental area during cueinduced reinstatement of cocaine seeking in rats. J Neurosci. 2012; 32:13309-13325. [PubMed: 22993446]

Mahler SV, Smith RJ, Moorman DE, Sartor GC, Aston-Jones G. Multiple roles for orexin/hypocretin in addiction. Prog Brain Res. 2012; 198:79-121. [PubMed: 22813971] 
Marcus JN, Aschkenasi CJ, Lee CE, Chemelli RM, Saper CB, Yanagisawa M, Elmquist JK. Differential expression of orexin receptors 1 and 2 in the rat brain. J Comp Neurol. 2001; 435:625. [PubMed: 11370008]

McElhinny CJ, Lewin AH, Mascarella SW, Runyon S, Brieaddy L, Carroll FI. Hydrolytic instability of the important orexin 1 receptor antagonist SB-334867: possible confounding effects on in vivo and in vitro studies. Bioorg Med Chem Lett. 2012; 22:6661-6664. [PubMed: 23031594]

Moorman DE, Aston-Jones G. Orexin-1 receptor antagonism decreases ethanol consumption and preference selectively in high-ethanol-preferring Sprague-Dawley rats. Alcohol. 2009; 43:379_ 386. [PubMed: 19671464]

Moorman DE, Aston-Jones G. Orexin/hypocretin modulates response of ventral tegmental dopamine neurons to prefrontal activation: diurnal influences. J Neurosci. 2010; 30:15585-15599. [PubMed: 21084614]

Morganstern I, Chang GQ, Barson JR, Ye Z, Karatayev O, Leibowitz SF. Differential effects of acute and chronic ethanol exposure on orexin expression in the perifornical lateral hypothalamus. Alcohol Clin Exp Res. 2010; 34:886-896. [PubMed: 20331576]

Mukai K, Kim J, Nakajima K, Oomura Y, Wayner MJ, Sasaki K. Electrophysiological effects of orexin/hypocretin on nucleus accumbens shell neurons in rats: an in vitro study. Peptides. 2009; 30:1487-1496. [PubMed: 19416746]

Muschamp JW, Hollander JA, Thompson JL, Voren G, Hassinger LC, Onvani S, Kamenecka TM, Borgland SL, Kenny PJ, Carlezon WA Jr. Hypocretin (orexin) facilitates reward by attenuating the antireward effects of its cotransmitter dynorphin in ventral tegmental area. Proc Natl Acad Sci. 2014; 111:E1648-E1655. [PubMed: 24706819]

Nocjar C, Zhang J, Feng P, Panksepp J. The social defeat animal model of depression shows diminished levels of orexin in mesocortical regions of the dopamine system, and of dynorphin and orexin in the hypothalamus. Neuroscience. 2012; 218:138-153. [PubMed: 22626650]

Patyal R, Woo EY, Borgland SL. Local hypocretin-1 modulates terminal dopamine concentration in the nucleus accumbens shell. Front Behav Neurosci. 2012; 6:82. [PubMed: 23226119]

Rhodes JS, Best K, Belknap JK, Finn DA, Crabbe JC. Evaluation of a simple model of ethanol drinking to intoxication in C57BL/6J mice. Physiol Behav. 2005; 84:53-63. [PubMed: 15642607]

Rhodes JS, Ford MM, Yu CH, Brown LL, Finn DA, Garland T Jr, Crabbe JC. Mouse inbred strain differences in ethanol drinking to intoxication. Genes Brain Behav. 2007; 6:1-18. [PubMed: 17233637]

Sakurai T, Amemiya A, Ishii M, Matsuzaki I, Chemelli RM, Tanaka H, Williams SC, Richardson JA, Kozlowski GP, Wilson S, Arch JR, Buckingham RE, Haynes AC, Carr SA, Annan RS, McNulty DE, Liu WS, Terrett JA, Elshourbagy NA, Bergsma DJ, Yanagisawa M. Orexins and orexin receptors: a family of hypothalamic neuropeptides and $\mathrm{G}$ protein-coupled receptors that regulate feeding behavior. Cell. 1998; 92:573-585. [PubMed: 9491897]

Schneider ER, Rada P, Darby RD, Leibowitz SF, Hoebel BG. Orexigenic peptides and alcohol intake: differential effects of orexin, galanin, and ghrelin. Alcohol Clin Exp Res. 2007; 31:1858-1865. [PubMed: 17850217]

Sharma R, Sahota P, Thakkar MM. Role of adenosine and the orexinergic perifornical hypothalamus in sleep-promoting effects of ethanol. Sleep. 2014; 37:525-533. [PubMed: 24587575]

Shoblock JR, Welty N, Aluisio L, Fraser I, Motley ST, Morton K, Palmer J, Bonaventure P, Carruthers NI, Lovenberg TW, Boggs J, Galici R. Selective blockade of the orexin-2 receptor attenuates ethanol self-administration, place preference, and reinstatement. Psychopharmacology. 2011; 215:191-203. [PubMed: 21181123]

Sparta DR, Sparrow AM, Lowery EG, Fee JR, Knapp DJ, Thiele TE. Blockade of the corticotropin releasing factor type 1 receptor attenuates elevated ethanol drinking associated with drinking in the dark procedures. Alcohol Clin Exp Res. 2008; 32:259-265. [PubMed: 18162072]

Srinivasan S, Simms JA, Nielsen CK, Lieske SP, Bito-Onon JJ, Yi H, Hopf FW, Bonci A, Bartlett SE. The dual orexin/hypocretin receptor antagonist, almorexant, in the ventral tegmental area attenuates ethanol self-administration. PLoS ONE. 2012; 7:e44726. [PubMed: 23028593]

Voorhees CM, Cunningham CL. Involvement of the orexin/hypocretin system in ethanol conditioned place preference. Psychopharmacology. 2011; 214:805-818. [PubMed: 21107540] 
Winsky-Sommerer R, Yamanaka A, Diano S, Borok E, Roberts AJ, Sakurai T, Kilduff TS, Horvath $\mathrm{TL}$, de Lecea L. Interaction between the corticotropin-releasing factor system and hypocretins (orexins): a novel circuit mediating stress response. J Neurosci. 2004; 24:11439-11448. [PubMed: 15601950]

Wise RA. Addictive drugs and brain stimulation reward. Annu Rev Neurosci. 1996; 19:319-340. [PubMed: 8833446]

Yoshida K, McCormack S, España RA, Crocker A, Scammell TE. Afferents to the orexin neurons of the rat brain. J Comp Neurol. 2005; 494:845-861. [PubMed: 16374809] 

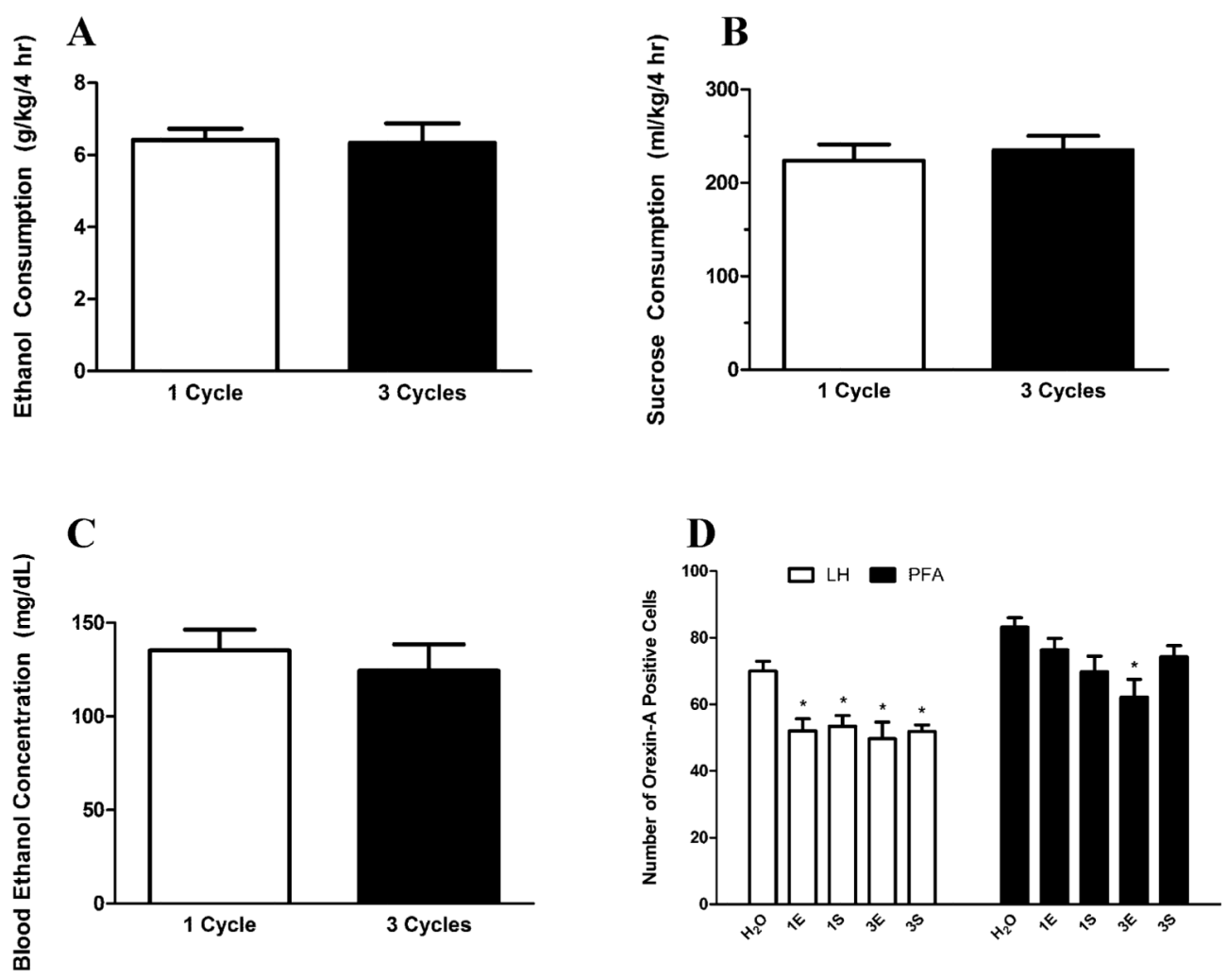

Fig. 1.

Comparison of binge-like drinking behavior and hypothalamic orexin $(\mathrm{OX})$ levels between mice exposed to 1 or 3 cycles of drinking-in-the-dark (DID). Mice with access to ethanol $(\mathrm{EtOH})$ bottles drank comparable levels of $\mathrm{EtOH}$ on the final day of testing regardless of whether they experienced 1 or 3 cycles of DID (A). Similarly, mice that experienced 1 or 3 cycles of DID consumed equivalent levels of sucrose $(\mathbf{B})$. No differences in blood EtOH concentrations were observed in the mice that experienced either 1 or 3 cycles of binge-like EtOH drinking (C). Assessment of hypothalamic OX levels in these mice (D) revealed that, relative to animals with access to water, binge-like EtOH drinking caused a reduction in OX levels in the lateral hypothalamus ( $\mathrm{LH})$ of animals that experienced 1 or 3 cycles of EtOH as well as 1 or 3 cycles of sucrose. However, in the PFA, only animals that experienced 3 cycles of EtOH displayed significant reductions in OX levels, while those that experienced 1 cycle of EtOH as well as 1 or 3 cycles of sucrose exhibited no alterations in OX immunoreactivity (IR) in the PFA. LH, lateral hypothalamus; PFA, perifornical area of the hypothalamus; $\mathrm{H}_{2} \mathrm{O}$, water group; 1E, 1-cycle $\mathrm{EtOH}$ group; 3E, 3-cycle EtOH group; 1S, 1cycle sucrose group; $3 \mathrm{~S}, 3$-cycle sucrose group; *denotes $p<0.05$ relative to $\mathrm{H}_{2} \mathrm{O}$ group in the same region. Data are presented as mean \pm SEM. 


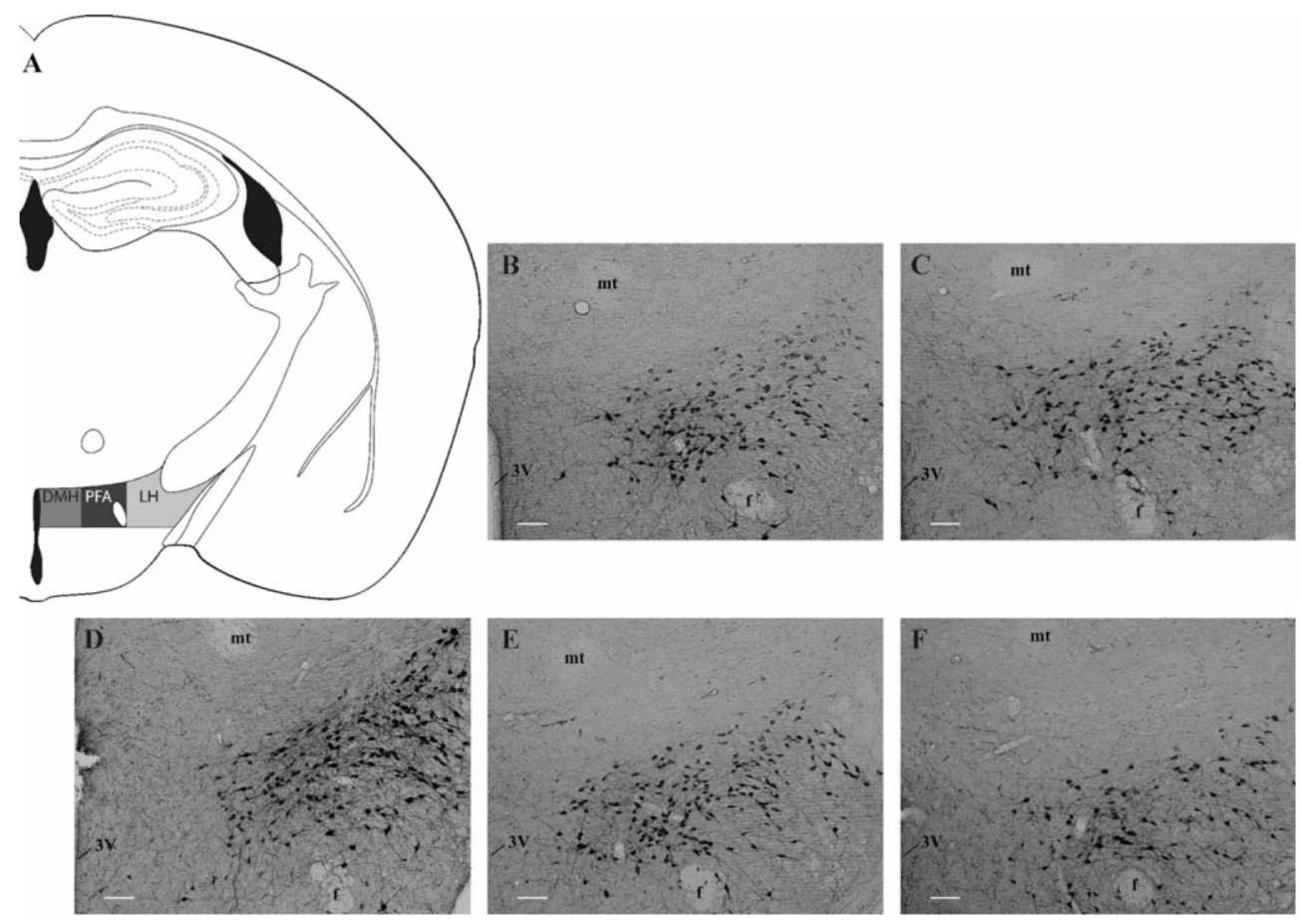

Fig. 2.

Binge-like ethanol (EtOH) drinking reduces hypothalamic orexin (OX) levels. The subregions of the hypothalamus investigated includes the LH, PFA, and DMH (A), photomicrographs from the 1-cycle EtOH (B), 3-cycle EtOH (C), water (D), 1-cycle sucrose $(\mathbf{E})$, and 3-cycle sucrose (F) groups representing qualitative changes in OX expression. White scale bar in $\mathrm{B}-\mathrm{F}=10.0 \mu \mathrm{m}$. 3V, third ventricle; $\mathrm{DMH}$, dorsomedial hypothalamus; $\mathrm{f}$, fornix; LH, lateral hypothalamus; mt, mammillothalamic tract; PFA, perifornical area of the hypothalamus. 
A
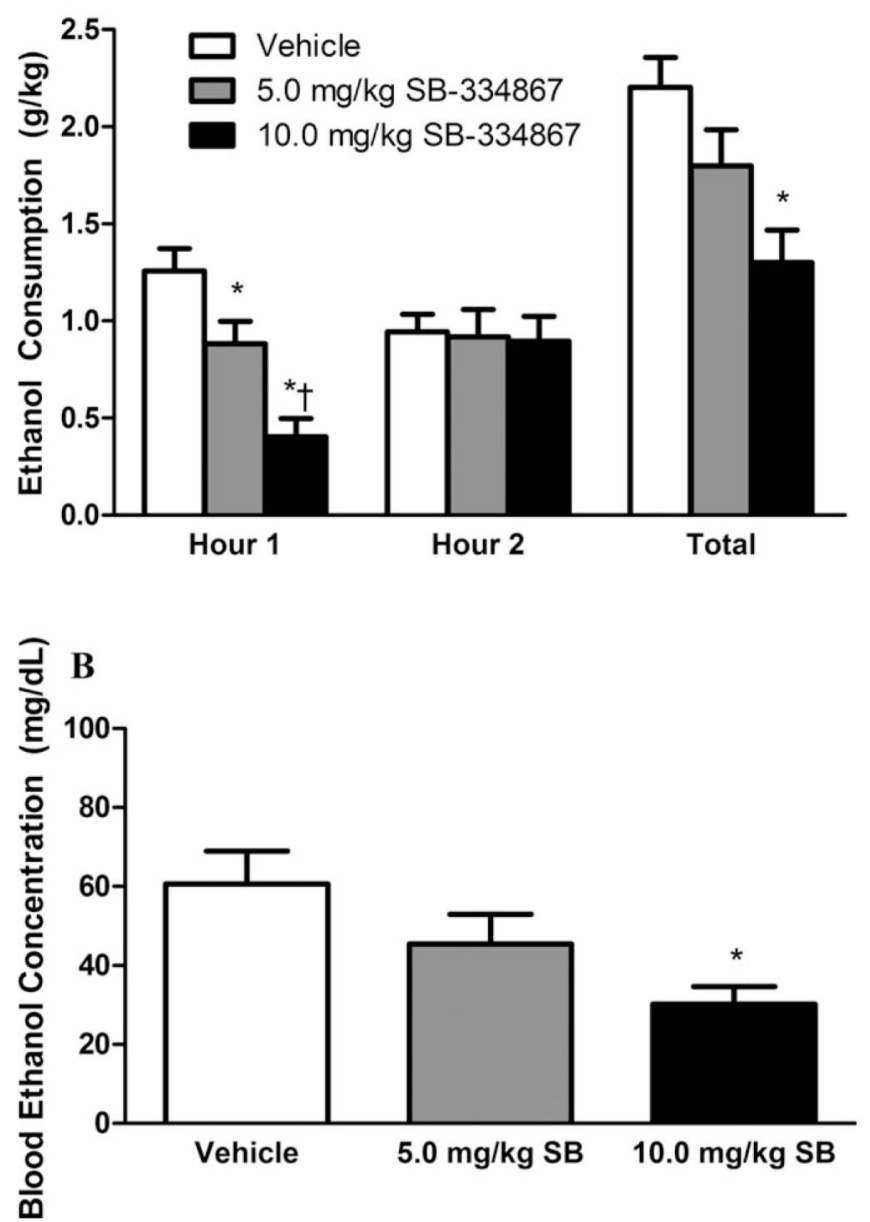

Fig. 3.

Pharmacological inhibition of the orexin-1 receptor reduces excessive ethanol (EtOH) consumption and blood EtOH concentration (BEC). Both the $5.0 \mathrm{mg} / \mathrm{kg}$ and $10.0 \mathrm{mg} / \mathrm{kg}$ doses of SB-334867 (SB) protected against excessive EtOH consumption during the first hour of testing relative to vehicle-treated controls; however, only the $10.0 \mathrm{mg} / \mathrm{kg}$ dose caused a significant reduction in EtOH drinking across the full 2-hour test period (A). Prior treatment with the $10.0 \mathrm{mg} / \mathrm{kg}$ dose of $\mathrm{SB}$ caused a significantly lower BEC relative to vehicle-treated controls $(\mathbf{B}) .{ }^{*}$ denotes $p<0.05$ relative to vehicle group at the same time point; $\dagger$ denotes $p<.05$ relative to $5.0 \mathrm{mg} / \mathrm{kg}$ group at the same time point. Data are presented as mean \pm SEM. 


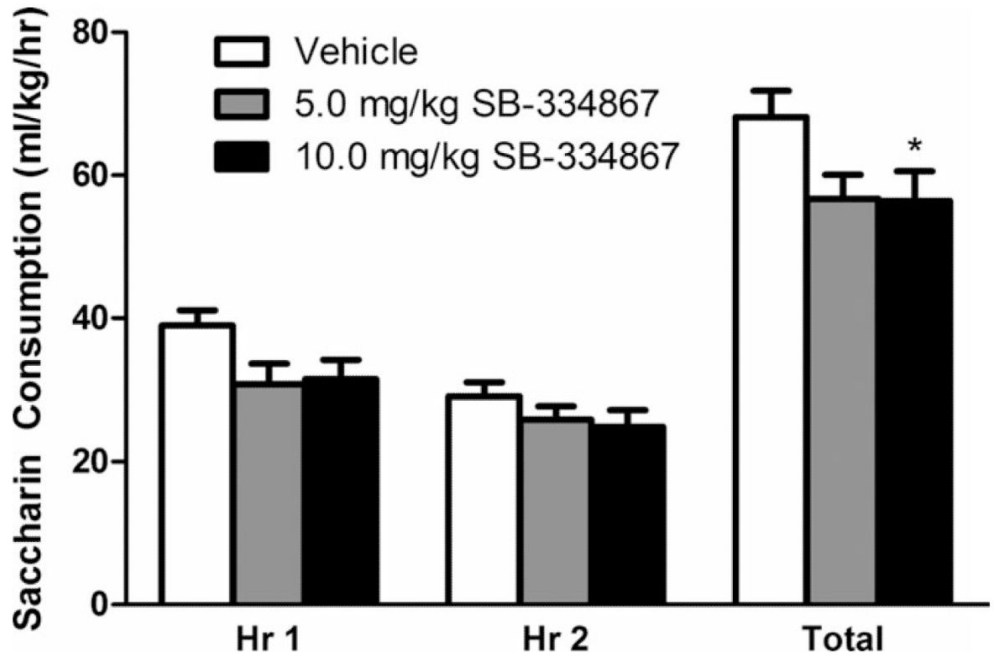

Fig. 4.

Pharmacological inhibition of the orexin-1 receptor reduces excessive saccharin consumption. Both doses of SB significantly reduced excessive saccharin drinking over the 2 hours of drinking-in-the-dark testing. This effect appears to be driven by the blunting of saccharin intake during the first, but not second hour of testing. * denotes $p<0.05$ relative to vehicle group at the same time point. Data are presented as mean \pm SEM. 


\section{皇}

ํํํ

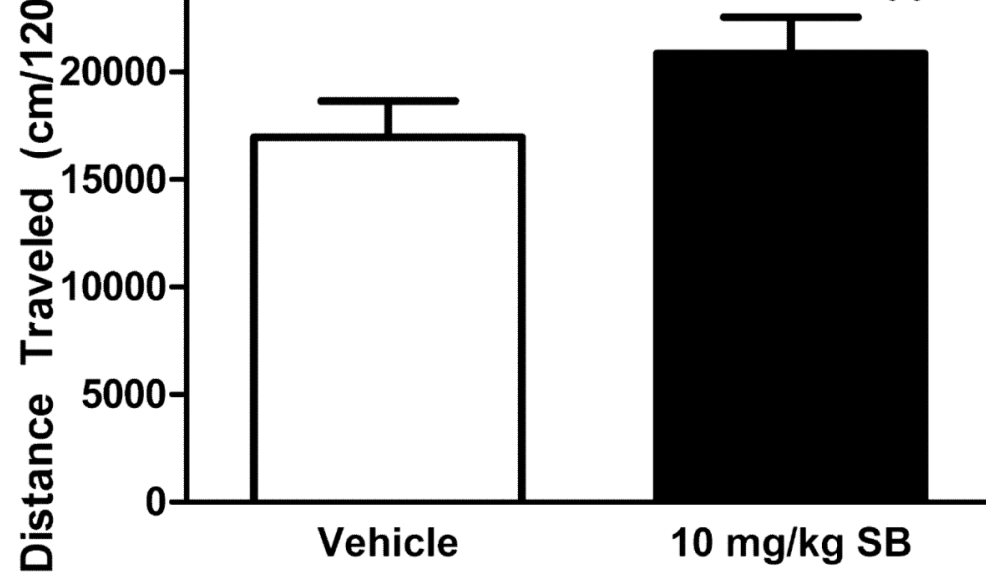

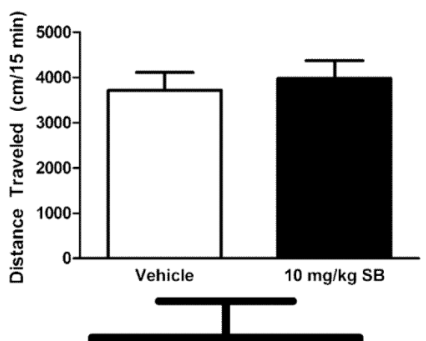

2000

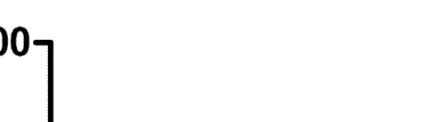

Fig. 5.

Treatment with SB-334867 (SB) does not affect locomotor activity. The $10.0 \mathrm{mg} / \mathrm{kg}$ dose of SB had no discernable effects on locomotor activity across the full 2 hours of testing nor did it have any immediate effects during the first 15 minutes of locomotor testing (inset). Data are presented as mean \pm SEM. 